\title{
MASS PREDICTIONS FROM THE GARVEY-KELSON MASS RELATIONS*
}

\author{
J. JÄNECKE and P. J. MASSON \\ Department of Physics, University of Michigan \\ Ann Arbor, Michigan 48109
}

Part A: The transverse Garvey-Kelson mass relation represents a homogeneous third-order partial difference equation. Procedures are described for estimating masses of nuclei with $N \geqslant Z$ from the most general solution of this difference equation subject to a $\chi^{2}$ minimization, using the recent atomic mass adjustment of Wapstra, Audi, and Hoekstra as a boundary condition. A judicious division of the input data into subsets of neutron-rich and proton-rich nuclei had to be introduced to reduce systematic errors in long-range extrapolations. Approximately $\mathbf{5 6 0 0}$ mass-excess values for nuclei with $2 \leqslant Z \leqslant 103,4 \leqslant N \leqslant 157$, and $N \geqslant Z$ (except $N=Z=$ odd for $A<40$ ) have been calculated. The standard deviation for reproducing the known mass-excess values is $\sigma_{\mathrm{m}} \approx 103 \mathrm{keV}$.

Part B: The charge-symmetric mass relation of Kelson and Garvey for estimating masses of nuclei with $Z>N$ and $T \geqslant 1$ is described. The calculations make use of the experimental Coulomb energy differences between $T=\frac{1}{2}$ mirror nuclei for $A \leqslant 59$ and of estimated values for $A>59$. Some 250 mass-excess values have been calculated. The standard deviation for reproducing the known values is $\sigma_{\mathrm{m}} \approx 231 \mathrm{keV}$. $\odot 1988$ Academic Press, Inc.

\footnotetext{
* Supported in part by the U.S. National Science Foundation and the U.S.-Israel Binational Science Foundation
}

0092-640X/88 $\$ 3.00$

Copyright (C) 1988 by Academic Press, Inc.

All rights of reproduction in any form reserved. 


\section{CONTENTS}

PART A: NUCLEI WITH $N \geqslant Z \ldots \ldots \ldots \ldots \ldots \ldots \ldots \ldots$

The Transverse Garvey-Kelson Relation $\ldots \ldots \ldots \ldots \ldots \ldots, 266$

Method of Mass Prediction ................. 267

PART B: NUCLEI WITH $Z>N$ AND $T \geqslant 1 \ldots \ldots \ldots \ldots .270$

The Kelson-Garvey Relation $\ldots \ldots \ldots \ldots \ldots \ldots \ldots \ldots .270$

Method of Mass Prediction ................. 270

\section{PART A: NUCLEI WITH $N \geqslant Z$}

\section{The Transverse Garvey-Kelson Relation}

The ground-state energies $M(N, Z)$ of a system of $A$ nucleons ( $N$ neutrons and $Z$ protons) can in principle be obtained from the general solutions of the manybody problem, making use of the nuclear many-body Hamiltonian. Approximations, assumptions, or models must be introduced for any practical application. Alternately, one may consider whether differences $M(N$ $+\Delta N, Z+\Delta Z)-M(N, Z)$ can be understood on the basis of more limited information about the nuclear many-body system. In particular, one may construct a homogeneous difference equation

$$
\sum_{i=1}^{\alpha} C_{i} M\left(N+\Delta N_{i}, Z+\Delta Z_{i}\right) \approx 0
$$

with small integer coefficients $C_{i}(= \pm 1, \pm 2, \cdots)$ and a small number of terms $\alpha$, for arbitrary values of $N$ and $Z$. Such an equation, if approximately satisfied, can be used as a recursion relation to estimate ground-state binding energies of nuclei if only one of the experimental mass values in Eq. (1) is unknown. Repeated applications of Eq. (1) permit long-range extrapolations.

Equation (1) may also be viewed as a partial difference equation in the variables $N$ and $Z$. The general solution of this difference equation can be used to construct a mass equation with the known experimental masses as a boundary condition. Usually, the number of known masses exceeds the number of adjustable parameters, and an appropriate $\chi^{2}$-minimization procedure is therefore indicated.
The transverse Garvey-Kelson relation ${ }^{1,2}$ was introduced about 20 years ago as a tool for predicting masses of unknown neutron-rich and proton-rich nuclei. It is the simplest nontrivial homogeneous mass relation and can be written as

$$
\begin{array}{r}
M(N+2, Z-2)-M(N, Z)+M(N+1, Z) \\
-M(N+2, Z-1)+M(N, Z-1) \\
-M(N+1, Z-2) \approx 0 .
\end{array}
$$

It is displayed schematically in Fig. 1.

The most direct heuristic proof of Eq. (1) follows from an independent-particle picture with fourfold-degenerate Hartree-Fock or Nilsson-like single-particle levels. Figure 2a displays a representation of Eq. (2), and it can be seen that single-particle energies, as well as effective interactions between proton-proton, neutronneutron, and neutron-proton pairs, cancel out in this model. One has to assume, though, that these quantities vary slowly and smoothly with nucleon number $A$. The relation cannot be used to cross the $N=Z$ line, and the cancellation of terms is not valid if one member in Eq. (2) is a self-conjugate nucleus with $N=Z=$ odd. This behavior is displayed in Fig. $2 b$, where, unlike Fig. 2a, the effective neutron-proton interaction energies involve particles from different orbits and do not cancel.

The relation is also supported by simple shellmodel mass equations. ${ }^{2,3}$ Assuming that neutrons and protons occupy the same $j$ shell, Eq. (2) is satisfied both in the seniority coupling scheme and with isospin as a good quantum number. Equation (2) is also satisfied approximately in the liquid drop model. ${ }^{4}$ 


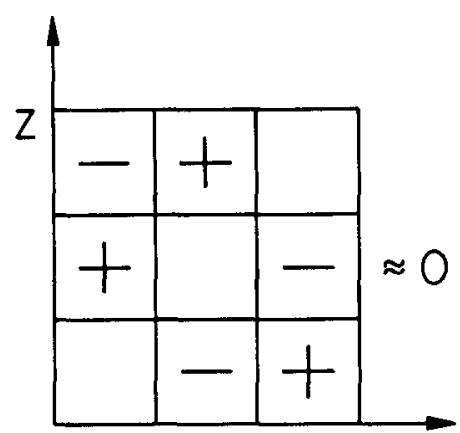

N

portantly, one has to decide which region of nuclei to use as data base for determining the functions $G_{i}(k)$. Updated sets of mass predictions based on all the then available mass data appeared over 10 years ago. ${ }^{6}$

Only recently have difficulties with long-range extrapolations established the need for inhomogeneous source terms. ${ }^{5,7}$ The main systematic effects are associated with higher-order perturbations in isospin. Mass predictions based on the homogeneous equations [Eq. (2) or (3)] and the simultaneous use of all available mass data must therefore be treated with caution.

\section{Method of Mass Prediction}

mass relation. The boxes represent nuclei from the nuclidic chart, and the plus and minus signs indicate that the respective mass values have to be added or subtracted.

The relation inaccuracy of Eq. (2), defined as the deviation of the left-hand side from zero, was found ${ }^{5}$ to be $(+15 \pm 222) \mathrm{keV}$ based on 809 mass combinations. The relation is better satisfied in heavier nuclei than in lighter ones.

Viewing Eq. (2) as a homogeneous third-order partial difference equation yields $\mathrm{s}^{2,3}$

$$
M(N, Z)=G_{1}(N)+G_{2}(Z)+G_{3}(N+Z)
$$

as the most general solution satisfying the difference equation. The mass parameters $G_{1}(N), G_{2}(Z)$, and $G_{3}(A)$ are arbitrary point functions of their arguments. Equation (3) is valid for nuclei with $N>Z$ and $N=Z$ $=$ even. Coulomb energies are not well described ${ }^{2}$ by Eq. (3). The functions can be constructed uniquely from a $\chi^{2}$ minimization of the differences $M(N, Z)-M_{\text {exp }}(N$, $Z)$. Weight factors have to be introduced, but more im-

The present mass predictions make use of Eq. (3). The point functions $G_{i}(k)$ were obtained from a $\chi^{2}$-minimization procedure. The system of typically 450 linear equations in 450 unknown quantities is solved with a computer program which makes use of sparse matrix subroutines for manipulating nonsingular matrices with many vanishing matrix elements. The recent mass evaluation of Wapstra et al. ${ }^{8}$ served as a data base. Individual weight factors were introduced by quadratically adding $100 \mathrm{keV}$ to the experimental uncertainties. The added energy has the characteristics of an uncertainty of the theory. This leads to approximately equal weight factors for mass values with uncertainties less than 100 $\mathrm{keV}$. These weight factors were also used for calculating standard deviations.

As an example of the difficulties with long-range extrapolations, Fig. 3 displays the differences between experimental and calculated masses (see Refs. 5 and 7 for more details). Here, only nuclei with neutron excesses $E=N-Z$ in the range $E_{\text {stab }}-\frac{1}{2} W<E \leqslant E_{\text {stab }}$ $+\frac{1}{2} W$ with $E_{\text {stab }}=0.4 A^{2} /(200+A)$ near the line of $\beta$ stability were used to calculate the point functions $G_{i}(k)$.

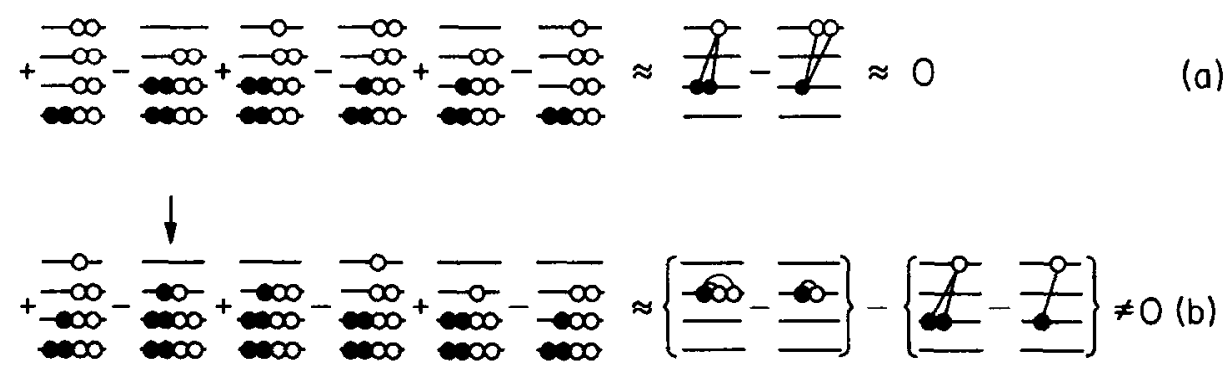

- proton

o neutron

Figure 2. Part (a) is a representation of the transverse mass relation based on an extreme single-particle picture. Part (b) shows that the relation is not satisfied when odd-odd self-conjugate nuclei are included. Here, terms with the effective neutron-proton interaction $I_{\mathrm{np}}$ involve particles from different orbits and do not cancel. 


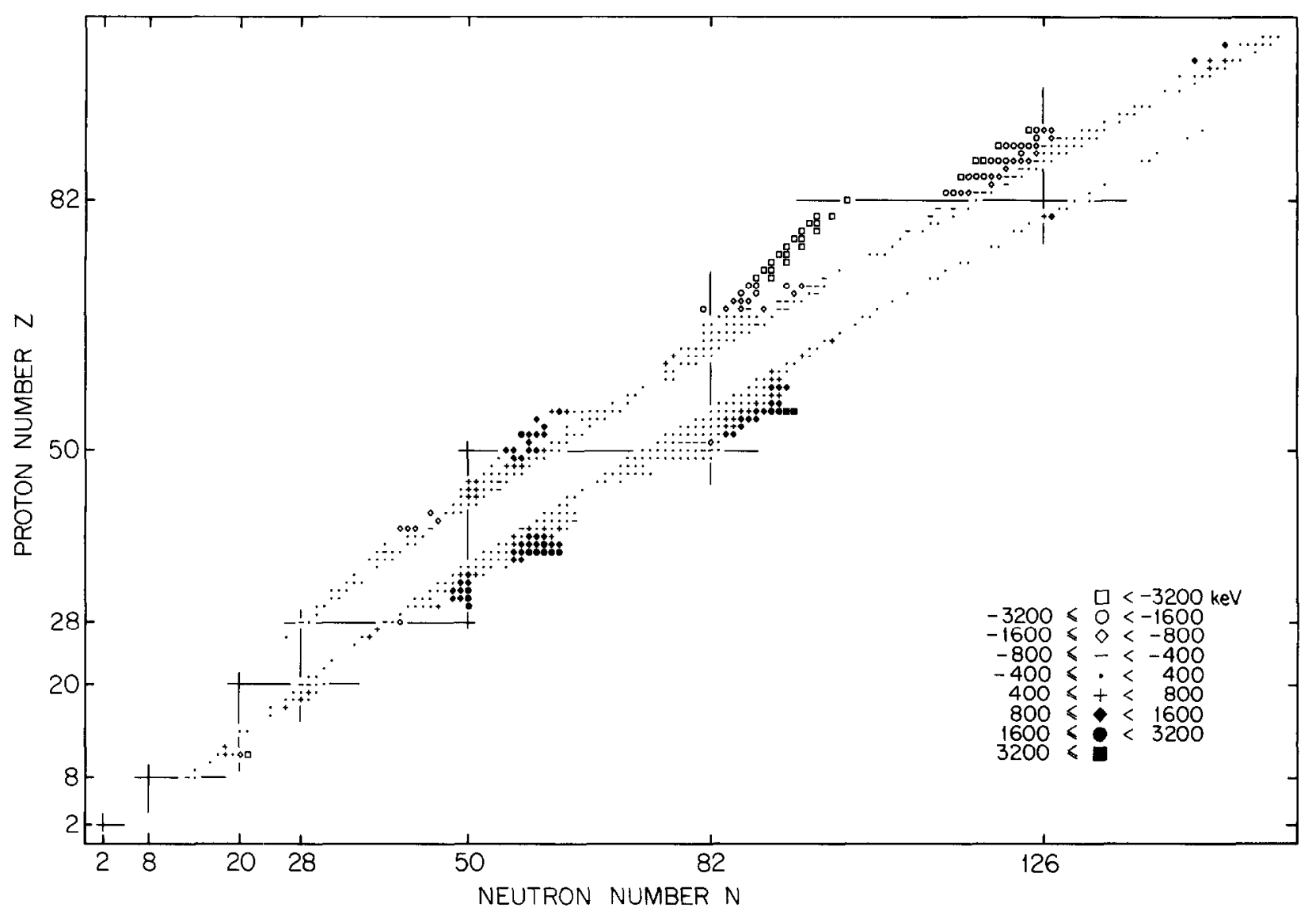

Figure 3. Differences between experimental and calculated masses from Eq. (3). The symbols represent nuclei with residuals in the indicated energy ranges. A subset of 980 nuclidic masses along the line of $\beta$ stability (not shown) was used to determine the mass parameters. The figure displays only residuals for extrapolated mass values.

Figure 3 is for $W=8$, and the 980 data points (not shown) are reproduced very well with a standard deviation of $\sigma_{\mathrm{m}}=79 \mathrm{keV}$. Only the residuals for the extrapolated masses are displayed in the figure. Systematic deviations can be seen clearly.

Separate adjustments for subsets of input data are needed to reduce systematic errors with long-range extrapolations. This greatly increases the number of adjustable parameters. The mass predictions of the present contribution were obtained by treating nuclei on the neutron-rich and proton-rich sides of the line of $\beta$ stability independently. However, a small overlap of input data became necessary to ensure data bases containing at least three input data points for each value of $A$. The adjustments were obtained using the regions

$$
R_{1}^{\prime}: E>E_{\text {stab }}-1 \text { for neutron-rich nuclei }
$$

and

$$
R_{2}^{\prime}: E \leqslant E_{\text {stab }}+1 \text { for proton-rich nuclei. }
$$

The residuals for these two adjustment regions are displayed in Figs. 4 and 5. Also included are extrapolations into the region of the other data base. This allows a judgment on the quality of extrapolations. In some regions deviations exceeding $800 \mathrm{keV}$ begin to appear for $E=E_{\text {stab }}-3$ (Fig. 4) and $E=E_{\text {stab }}+3$ (Fig. 5). Predictions for region $R_{1}$ with nuclei $E>E_{\text {stab }}$ based on $R_{1}^{\prime}$, and for region $R_{2}$ with nuclei $E \leqslant E_{\text {stab }}$ based on $R_{2}^{\prime}$, are included in the main table. The statistical data for the two $\chi^{2}$ minimizations are shown in Table A. The standard deviation for reproducing all input data of approximately 1550 mass values is $103 \mathrm{keV}$ obtained by combining the results for the two regions. The combined number of parameters for the two adjustments is large. 


\section{J. JÄNECKE and P. J. MASSON Garvey-Kelson Mass Relations}

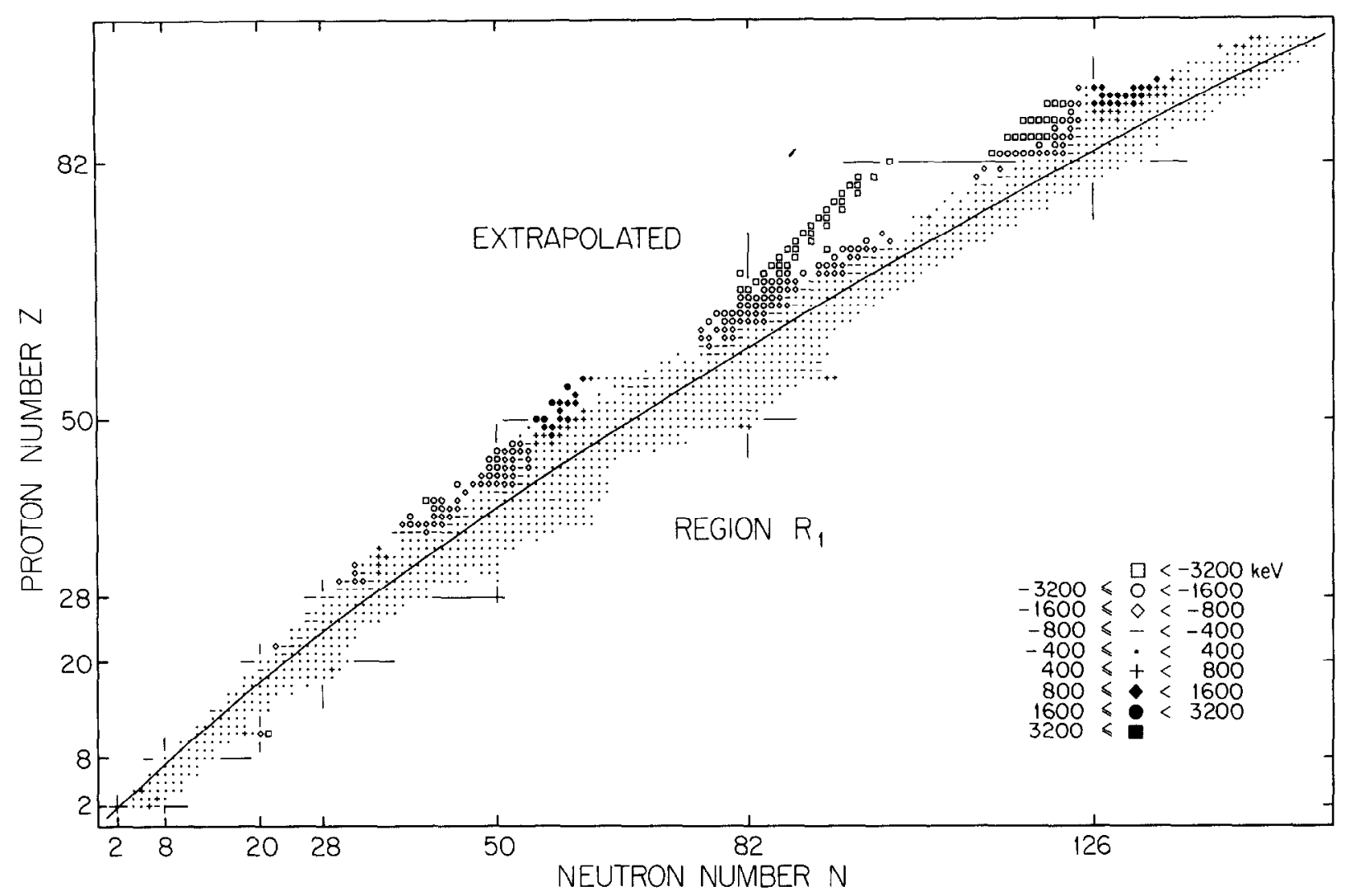

Figure 4. Differences between experimental and calculated masses from Eq. (3). The mass parameters were determined from the adjustment in region $R_{1}^{\prime}$ for nuclei on the neutron-rich side of the line of $\beta$ stability. The figure displays residuals for all known $N>Z$ and $N=Z=$ even nuclei.

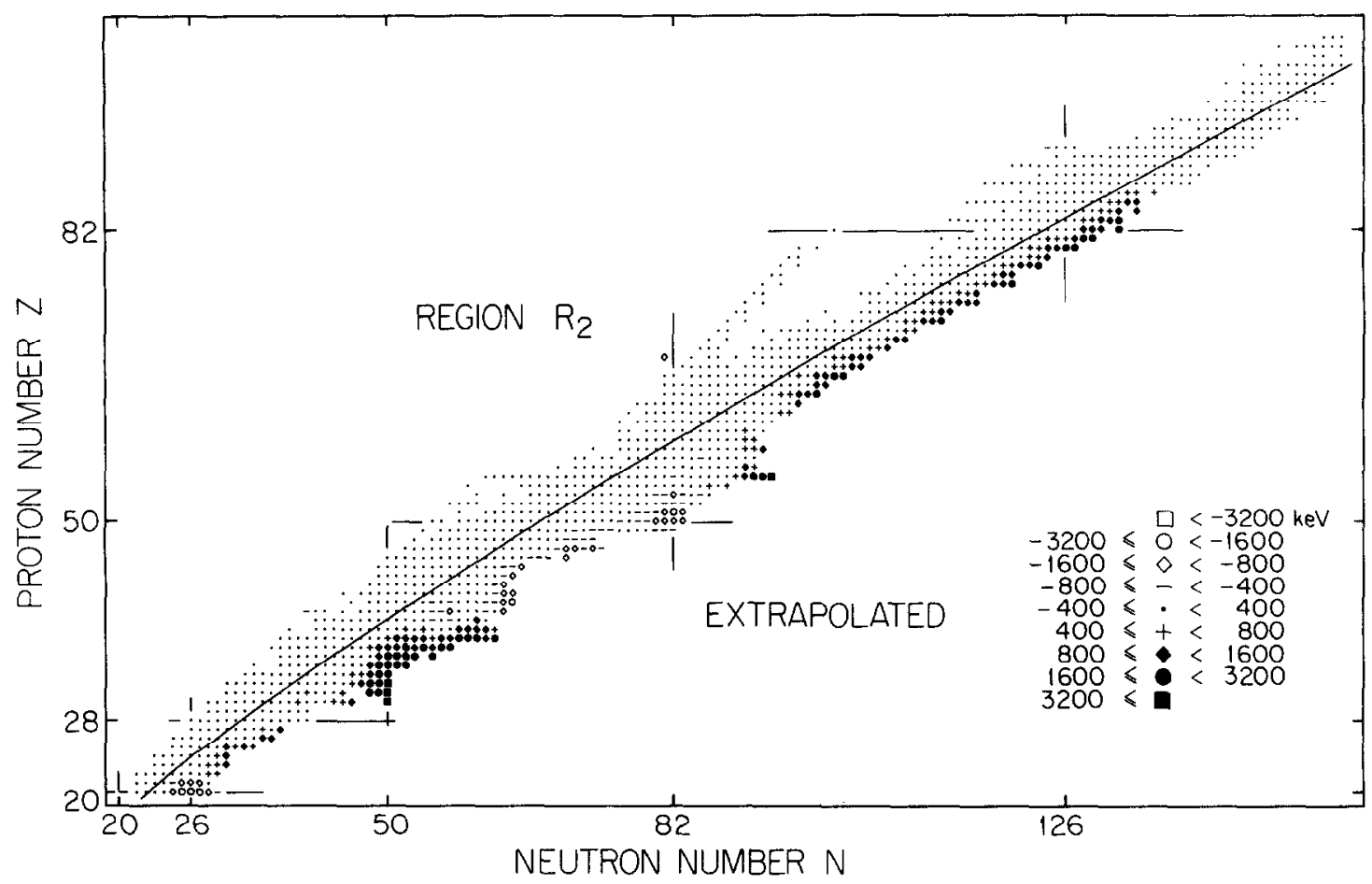

Figure 5. Differences between experimental and calculated masses from Eq. (3). The mass parameters were determined from the adjustment in region $R_{2}^{\prime}$ for nuclei on the proton-rich side of the line of $\beta$ stability. The figure displays residuals for all known $N>Z$ and $N=Z=$ even nuclei. 
TABLE A

Statistical Data for the $\chi^{2}$ Minimizations

\begin{tabular}{lrrrr}
\hline & \multicolumn{3}{c}{ Region } & All \\
\cline { 2 - 4 } & $R_{1}$ & $R_{2}$ & $S$ & nuclei \\
\hline$Z$ (min) & 2 & 4 & 3 & 2 \\
$Z(\max )$ & 97 & 103 & 30 & 103 \\
$N(\min )$ & 4 & 4 & 2 & 2 \\
$N(\max )$ & 154 & 157 & 29 & 157 \\
Number of input data & & & & \\
$\quad$ points & 774 & 772 & $90^{a}$ & 1636 \\
Number of parameters & 492 & 436 & 0 & 928 \\
$\quad$ Number of predicted & & & & \\
$\quad$ mass values & 3718 & 1940 & 250 & 5908 \\
Standard deviation $\sigma_{\mathrm{m}}(\mathrm{keV})$ & 113 & 92 & 231 & 114 \\
\hline
\end{tabular}

Note. Regions $R_{1}$ and $R_{2}$ are for nuclei with $N>Z$ and $N=Z$ $=$ even with $R_{1}$ on the neutron-rich and $R_{2}$ on the proton-rich side of the line of $\beta$ stability (Part A). Region $S$ is for nuclei with $Z>N$ (Part B).

${ }^{a}$ Number of known values to which predicted values are compared.

\section{PART B: NUCLEI WITH $Z>N$ AND $T \geqslant 1$}

\section{The Kelson-Garvey Relation}

Since nuclear forces are charge-symmetric, the binding energies between mirror nuclei differ essentially only in their Coulomb energies. The unknown mass of a proton-rich nucleus can therefore be predicted from the known mass of its neutron-rich mirror nucleus if the electrostatic Coulomb energy difference between them can be estimated. A simple and successful procedure has been introduced by Kelson and Garvey. ${ }^{9,2,3}$ It is a mass relation which connects masses of higher-order mirror nuclei with (ordinary) mirror nuclei near the $N=Z$ line,

$$
\begin{aligned}
& M\left(A, T_{z}=-T\right)-M\left(A, T_{z}=+T\right) \\
& \approx \sum_{\substack{j=-(2 T-1) \\
A+j=\text { odd }}}^{+(2 T-1)}\left[M\left(A+j, T_{z}=-\frac{1}{2}\right)-M\left(A+j, T_{z}=+\frac{1}{2}\right)\right] .
\end{aligned}
$$

Here, $T_{z}=\frac{1}{2}(N-Z)$ is the $z$ component of the isospin $T$. Equation (4) is schematically displayed in Fig. 6. The mass differences between the most-proton-rich and most-neutron-rich members of an isospin multiplet can hence be estimated from known mass differences between $T=\frac{1}{2}$ mirror nuclei. A heuristic proof of this relation can again be obtained from an independentparticle picture with fourfold-degenerate Hartree-Fock or Nilsson-like single-particle levels. Figure 7 (drawn for
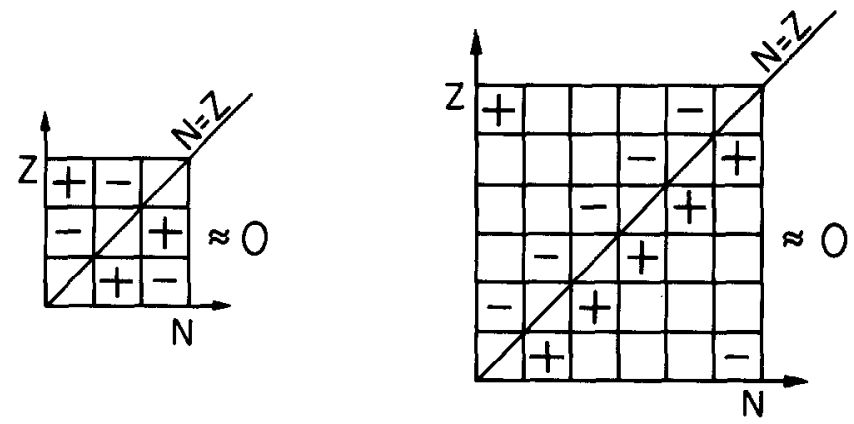

(a)

(b)

Figure 6. Schematic representation of the charge-symmetric KelsonGarvey relation (4) for two examples, $T=1$ (a) and $T=\frac{3}{2}$ (b).

$T=1$ ) shows that nuclear interactions cancel out, and Coulomb energies are approximately equal on both sides of the equation.

\section{Method of Mass Prediction}

The mass differences for the $T=\frac{1}{2}$ mirror nuclei up to $A=59$ were taken from the mass evaluation of Wapstra et al $^{8}$ Additional values up to $A=99$ were calculated from a Coulomb energy equation. ${ }^{10}$ Using Eq. (4) and the predictions of Part A for nuclei with $N$ $>Z$, mass predictions for approximately 250 protonrich nuclei up to $T_{z}=-3$ were made and combined in the main table of this issue with those from the first part. The statistical information is included in Table A. Good agreement with 90 known mass-excess values was achieved with a standard deviation of approximately $230 \mathrm{keV}$. However, systematic deviations of up to several hundred $\mathrm{keV}$ were also observed for those very-proton-rich nuclei which are unbound or only weakly bound with regard to the emission of protons or proton
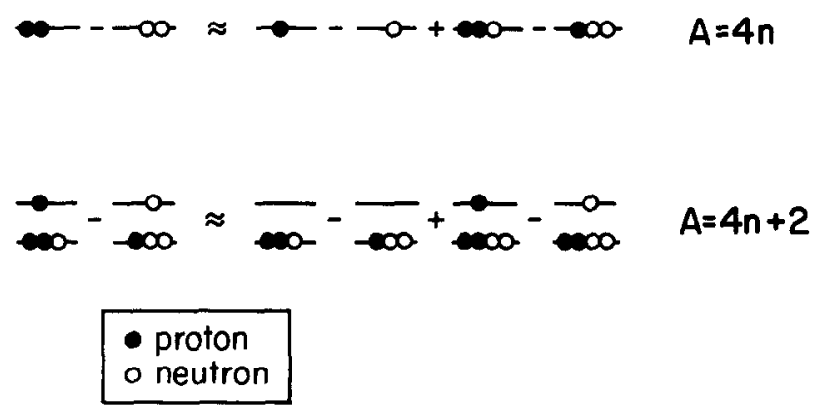

Figure 7. Representation of the charge-symmetric Kelson-Garvey relation for $T=1$ based on an extreme single-particle picture. 
pairs. This energy shift (Thomas-Ehrman shift) is the result of a Coulomb perturbation in the wave function.

\section{References}

1. G. T. Garvey and I. Kelson, Phys. Rev. Lett. 16, 197 (1966)

2. G. T. Garvey, W. J. Gerace, R. L. Jaffe, I. Talmi, and I. Kelson, Rev. Mod. Phys,. 41, S1 (1969)

3. G. T. Garvey, Annu. Rev. Nucl. Sci. 19, 433 (1969)

4. J. Jänecke and H. Behrens, Z. Phys. 256, 236 (1972); Phys. Rev. C 9, 1276 (1974)

5. J. Jänecke and E. Comay, Nucl. Phys. A 436, 108 (1985)
6. J. Jänecke, ATOMIC DATA AND NUClEAR Data TABLES 17, 455 (1976); E. Comay and I. Kelson, Atomic Data and Nuclear Data Tables 17, $463(1976)$

7. J. Jänecke, in Proceedings, 7th International Conference on Atomic Masses and Fundamental Constants (AMCO-7), edited by O. Klepper (Darmstadt 1984), p. 420

8. A. H. Wapstra, G. Audi, and R. Hoekstra, AToMIC DATA AND NuCleAR DATA TABLES 39, 281 (1988)

9. I. Kelson and G. T. Garvey, Phys. Lett. 23, 689 (1966)

10. E. Comay and J. Jänecke, Nucl. Phys. A 410, 103 (1983); J. Jänecke and E. Comay, Phys. Lett. B 140, 1 (1984) 